\title{
AUTOMATION AND QUEUE MANAGEMENT FOR NEO SURVEYING AND FOLLOW-UP
}

\author{
Alex R. Gibbs ${ }^{1}$, E. C. Christensen ${ }^{1}$, D. C. Fuls ${ }^{1}$, A. D. Grauer ${ }^{1}$, J. A. Johnson ${ }^{1}$, R. A. Kowalski ${ }^{1}$, \\ S. M. Larson ${ }^{1}$, G. J. Leonard ${ }^{1}$, R. G. Matheny ${ }^{1}$, R. L. Seaman ${ }^{1}$, and F. C. Shelly ${ }^{1}$
}

\begin{abstract}
The Catalina Sky Survey (CSS) at the University of Arizona operates three telescopes full-time in the search for near-Earth objects (NEOs). CSS has discovered 47\% of all known NEOs, reported over 46 million asteroid positions, and our photometry has been used to generate light curves for half a billion sources. Our telescopes are capable of highly automated data acquisition and we are working on making one of them autonomous from target selection to reporting of high-confidence data. I cover various aspects of the high-level automation at CSS, with emphasis on the queue manager software, and give a brief overview of CSS and our results.
\end{abstract}

\section{RESUMEN}

El Rastreo de los Cielos de Catalina (Catalina Sky Survey) en la Universidad de Arizona opera tres telescopios a tiempo completo en la búsqueda de objetos cercanos a la Tierra (NEOs). CSS ha descubierto el $47 \%$ de todos los NEOs conocidos, reportado 46 millones de posiciones de asteroides, y nuestra fotometría se ha utilizado para generar curvas de luz para 500 mil millones de estrellas. Nuestros telescopios son capaces de adquirir datos de forma altamente automatizada y estamos trabajando para que uno de ellos sea autónomo desde la selección del objetivo hasta la elaboración de informes de datos de alta confianza. Se cubren varios aspectos de la automatización de alto nivel en CSS, con énfasis en el software gestor de colas, dando una breve descripción de CSS y exponiendo los resultados obtenidos.

Key Words: methods: observational — minor planets, asteroids: general — surveys

\section{CATALINA SKY SURVEY GOALS}

The primary goals of the Catalina Sky Survey are to:

- Discover near-Earth objects (NEOs), especially those $140 \mathrm{~m}$ and larger, as requested by NASA (Stokes et al. 2003). CSS has discovered $47 \%$ of all known NEOs (CNEOS 2018).

- Follow up recent NEO discoveries and known NEOs that need further observations to significantly lessen the uncertainty of their orbits. This prevents NEOs from being lost and allows their trajectories to be more precisely extrapolated into the future, which in some cases leads to ruling out the possibility of impact in the near term.

- Report astrometry and photometry for all the asteroids detected, including main-belt asteroids. This results in improved orbital elements for all asteroids, which in turn allows them to be quickly identified and ruled out as new candidates the next time they are detected. In addition, useful science may come from such observations.

- Archive all our data and provide it to others,

\footnotetext{
${ }^{1}$ Lunar and Planetary Laboratory, University of Arizona, 1629 E. University Blvd, Tucson, AZ 85721-0092 (agibbs@lpl.arizona.edu).
}

for example the Catalina Real-Time Transient Survey, which has used it to generate light curves for 500 million objects (CRTS 2017).

As can be seen in Figure 1, CSS has made substantial contributions to the known NEO population since 2004. In 2017, CSS made $48 \%$ of the NEO discoveries, more than any other survey (MPC 2018).

\section{TELESCOPES}

We currently use three telescopes full-time and one other telescope for a few nights a month. Two are used almost exclusively for surveying for NEOs while two are used for following up recent discoveries and various known NEOs. Table 1 shows the characteristics of our three dedicated telescopes.

\section{HIGH-LEVEL AUTOMATION OVERVIEW}

High-level automation is required to accomplish our goals, particularly the efficient following up of recent NEO discoveries. This is especially necessary given the increased volume of NEO discoveries from us and others (Figure 1), and given the limited number of follow-up resources around the world. It is important that the resources be used wisely.

We employ scripts to obtain and prioritize followup targets from various sources and filter them for 


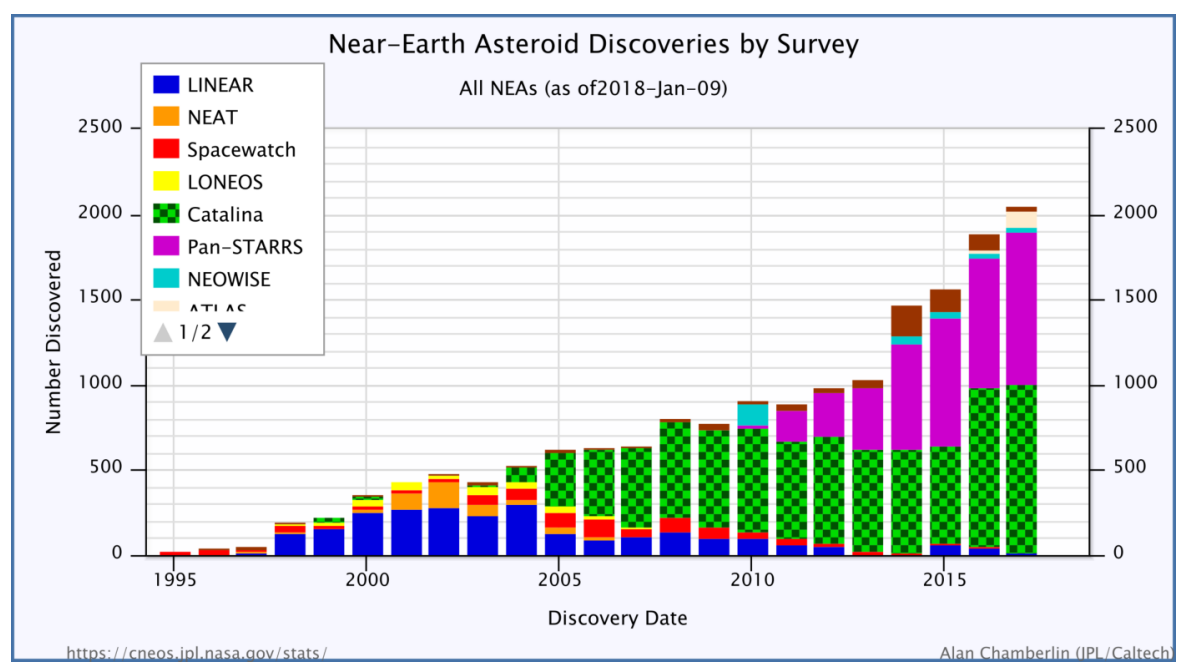

Fig. 1. Near-Earth asteroid discoveries by survey since 1995, showing CSS' contributions as checkered bars. Courtesy NASA/JPL-Caltech (CNEOS 2018).

TABLE 1

FULL-TIME CSS TELESCOPES

\begin{tabular}{cccccccc}
\hline \multicolumn{1}{c}{ Telescope } & $\begin{array}{c}\text { MPC } \\
\text { Code }\end{array}$ & f/\# & $\begin{array}{c}\text { Instrument } \\
(\mathrm{CCD})\end{array}$ & $\begin{array}{c}\text { FOV } \\
\left(\mathrm{deg}^{2}\right)\end{array}$ & Task & $\begin{array}{c}50 \% \text { Limiting } \\
\text { Mag. (V) }\end{array}$ & $\begin{array}{c}\text { Coverage } \\
\text { (per 10 hr.) }\end{array}$ \\
\hline 0.7 m Schmidt & 703 & $\mathrm{f} / 1.8$ & $10 \mathrm{k} \times 10 \mathrm{k}$ & 19.4 & Survey & 19.5 & $4,000 \mathrm{deg}^{2}$ \\
1.5 m Reflector & G96 & $\mathrm{f} / 1.6$ & $10 \mathrm{k} \times 10 \mathrm{k}$ & 5.0 & Survey & 21.3 & $1,000 \mathrm{deg}^{2}$ \\
1.0 m Cassegrain & I52 & $\mathrm{f} / 2.6$ & $2 \mathrm{k} \times 2 \mathrm{k}$ & 0.3 & Follow-Up & 21.5 & $50-80 \mathrm{NEOs}$ \\
\hline
\end{tabular}

our telescopes. Other software determines the best way to observe a particular target given its magnitude and rate and decide which telescope is best suited to observe the target. For example, we typically visit a moving target four times, but the software will decide whether each visit is to be a single exposure or multiple tracked exposures that are stacked together. The observer may adjust the automatic settings. The targets are then sent to the queue manager of a telescope, which will efficiently coordinate the observing of many targets throughout the night. Each of these steps is described in more detail below.

\section{DATA PROCESSING OVERVIEW}

Although not the main focus of this paper, a brief overview of our data processing is warranted. After images are acquired our pipeline performs flat fielding, source extraction using SExtractor ${ }^{2}$, and astrometric and photometric calibration using $\mathrm{SCAMP}^{3}$

\footnotetext{
${ }^{2}$ Bertin, E., \& Arnouts, S. 1996, A\&AS 117, 393

${ }^{3}$ Bertin, E. 2006, Astronomical Data Analysis Software and Systems XV, 351, 112
}

with the Gaia $\mathrm{DR} 1^{4}$ catalog. We do not use optical filters so we can maximize the quantity of astrometry. Image subtraction is used to find additional sources and they and the SExtractor sources are examined to find moving object candidates. Some candidates are identified as known objects and some are classified as unknown main-belt asteroids. The remaining candidates are sorted by their likelihood of being real and are validated by an observer. The data is archived on multiple mass storage units and consists of FITS images and flat data files. All moving object astrometry is sent to the Minor Planet Center where it is vetted and added to their database, which is accessible at their website.

\section{TARGETING}

Whether searching for new NEOs (survey mode) or extending the known arc of an asteroid's orbit (follow-up mode), the first thing to do is determine where to look or what needs to be followed up. Automated scripts aid us with these targeting decisions.

\footnotetext{
${ }^{4}$ Gaia Collaboration, Brown, A. G. A., Vallenari, A. et al. 2016, A\&A 595, A2
} 


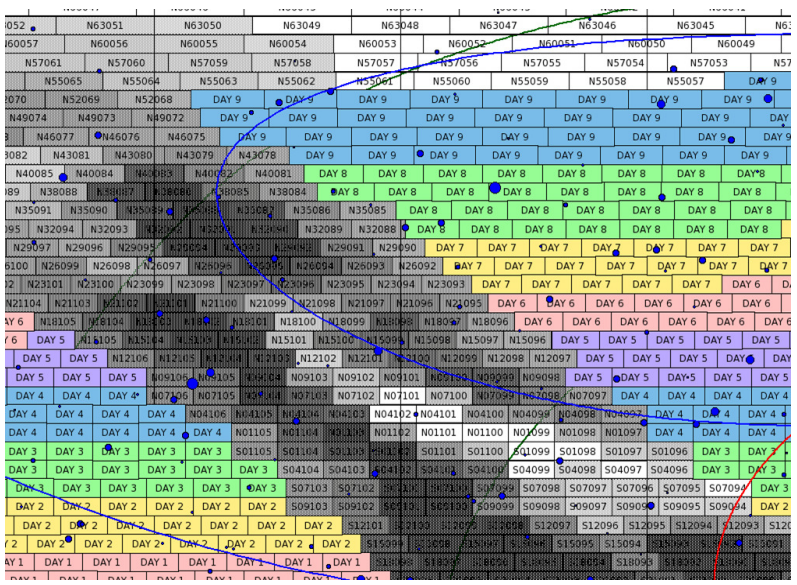

Fig. 2. Coverage tool used by observers to visualize and modify suvery plans, showing plans for multiple days that are avoiding the Milky Way (diagonal band).

\subsection{Survey Targeting}

We employ coverage planning software to systematically cover the sky in declination bands, though observers may override plans in the event of clouds, wind, etc. The planner takes into account areas of the sky that we want to avoid surveying, such as dense Milky Way regions and the area around the Moon. Our coverage tool (Figure 2) allows observers to view and modify plans, view recent sky coverage by our and other's telescopes, and visually monitor the progress of observations. Once the observer is happy with the plan it is submitted to the queue manager of the telescope. After that the observer has little interaction with the telescope and spends most of their time examining the NEO candidates presented by the data reduction pipeline.

\subsection{Follow-up Targeting}

The first step in doing NEO follow-up is figuring out what needs following up. We first compile a list of potential targets by looking at:

- New NEO candidates from that night

- The Minor Planet Center's NEO Confirmation Page

- Hazard assessment lists such as JPL's Scout

- Radar targets

- Potential space mission targets

- Known NEOs needing further observations

Some of these sources are checked multiple times per night since new discoveries are made all the time.

\subsection{Prioritizing Targets}

Prioritizing targets that need following up is more difficult than it sounds. For example, is it more important to follow up a relatively small asteroid that is buzzing by Earth and will soon be unobservable, or a large asteroid that is far away and visible for much longer? There are many factors to consider when deciding how urgently a NEO needs more observations. Our script looks at various characteristics of an asteroid candidate to calculate an overall priority, for example:

- Orbit category (NEO, PHA, main-belt)

- Size (absolute magnitude)

- Arc length and time last observed

- Magnitude trend (fading or brightening)

- Solar elongation trend

We are also working on a way to better determine if observing a target now will significantly reduce future uncertainty. If not then that will lower a target's current priority. The answer is not always obvious.

\subsection{Observability and Telescope Assignment}

Some telescopes are better suited to observe some targets than others. Deciding what targets are appropriate for a particular telescope involves looking at several telescope characteristics in relation to a given target:

- Magnitude and exposure limits vs. object's magnitude

- Declination range and altitude limits vs. object's location

- Field of view vs. object's ok-sky uncertainty

- Tracking and stacking ability vs. target's rate of motion

- Telescope availability (all night, limited time)

Since we have a range of telescopes at our disposal, we carefully consider the above items to decide where it is best to observe a target. We avoid doing follow-up on our survey telescopes unless absolutely necessary. However, some important targets require the wide field of view or deeper sky coverage of our $1.5 \mathrm{~m}$ survey telescope because covering the same area or going as deep with our $1 \mathrm{~m}$ followup telescope would take far too long. Weighing the cost of using one telescope against the cost of using a different one, and comparing that to the value of recovering a target, is a work in progress.

\subsection{Coordination}

In addition to coordinating follow-up efforts amongst our own telescopes, we and other follow-up telescopes need to coordinate our observations to:

- Prevent duplication of effort

- Ensure all important targets are observed

- Handle larger quantities of targets

- Automatically remove updated targets 


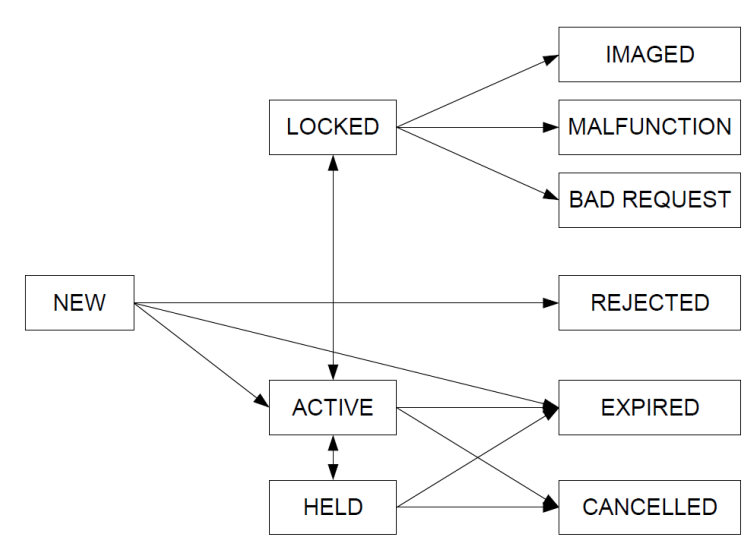

Fig. 3. Flow chart of the status of an image in the queue.

\section{QUEUE MANAGER}

In the past observers were only able to manually observe a few follow-up targets together, but that is not the most efficient use of telescope time. Because NEOs move at a variety of rates, the optimal time between successive exposures (cadence) varies from target to target, making it hard to manually schedule targets together. In addition, the large increase in NEO candidates and known NEOs over the last decade means that follow-up telescope time needs to be used wisely. This trend will only continue as more and larger surveys begin operating, such as Pan-STARRS2 and LSST.

Our queue management software (QMan) coordinates the observing of many targets of different priorities and cadences, taking into account all the observing parameters specified for each target submitted. QMan automatically adjusts the queue when targets are added or removed throughout the night. As new discoveries are made they need to be followed up and added to the queue, often at a higher priority than targets already on the queue, and if another telescope follows up a target before our telescope does then we may remove that target from our queue to reduce duplication of effort. QMan constantly adjusts the telescope scheduling to accommodate these and other changes so that we make efficient use of valuable telescope time.

\subsection{Data Structure}

At its lowest level, QMan automates the scheduling of individual exposures. However, a useful scientific observation often consists of multiple images. Sometimes it is also desirable to group related scientific observations together. QMan handles all these concepts:
- A single exposure is an image.

- The minimum set of images that give a useful result are an observation.

- Observations that should be made together comprise a group.

For example, in the case of NEO surveying:

- An image is a single exposure of a specific field.

- An observation is all the images of the same field, which will be compared to search for moving objects.

- A group is a set of observations of adjacent fields that will be done together, primarily as an easy way to give them all a specific and regular cadence between images.

Other kinds of observations, such as NEO followup and time sequences, can be set up with this structure. We also implement track-and-stack moving object detection sequences with this architecture, where we visit a field 4 times, but upon each visit we take multiple images while tracking at the target's rate and direction. The images are then stacked together to produce one image for the visit that improves signal-to-noise and reduces the trailing of the stars or the target that occurs when doing a single long exposure of a moving target. The data structures of the QMan handle this more complicated request too.

\subsection{Observation Parameters}

Submissions to the queue manager may specify numerous observing parameters and constraints:

- Where and when: right ascension, declination, and the time at which the target is at those coordinates.

- Motion: the target's rate and direction of motion so that it's position can be extrapolated at other times. The real-time calculation of a target's position from orbital elements is also planned. Stationary targets simply specify a zero rate.

- Bias rates to use, if desired.

- Filter to use.

- Exposure duration.

- Observation time window: the earliest and latest times to observe the target. The earliest time for subsequent images can be relative to a previous image, which allows images to be taken at specified intervals or regular cadences.

- Altitude or airmass limits.

- A target category or named priority which is mapped internally to a numeric priority. This allows the relative priority of targets to be adjusted from night to night if desired.

- Other items for data management. 


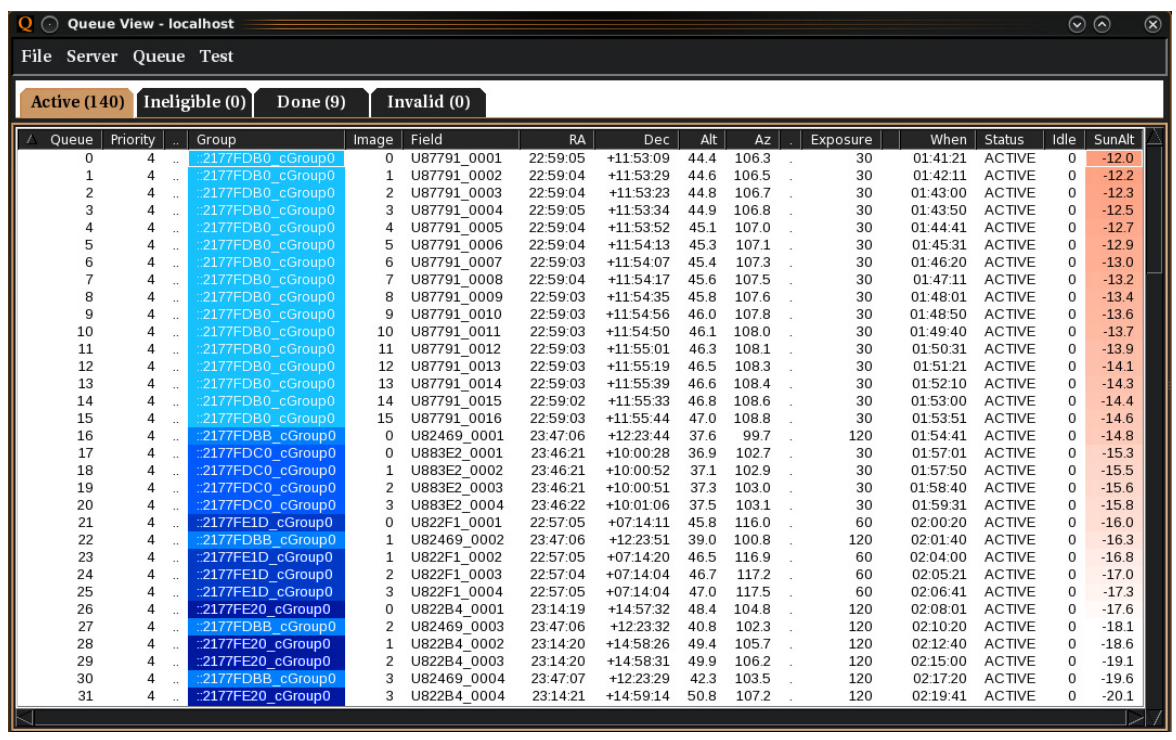

Fig. 4. Queue viewer (QView) used to monitor the status of targets on the queue and make adjustments if needed.

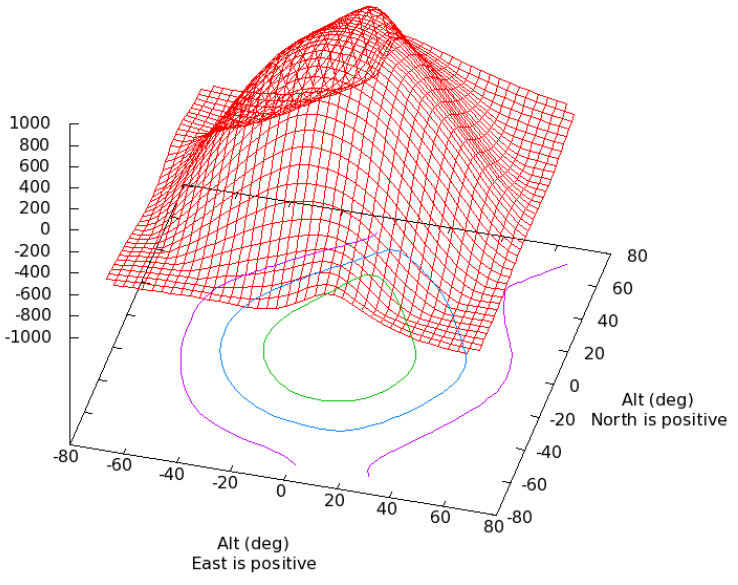

Fig. 5. Map of focus deviation, in motor steps, as a function of telescope altitude and azimuth at the CSS $1.5 \mathrm{~m}$ (G96). The map is used to automatically adjust focus with every telescope slew.

Submission clients are usually used to generate the properly formatted, low-level requests for specific kinds of targets and observations, such as survey, follow-up, track-and-stack, time series, etc.

\subsection{Internal Procedure}

Our queue manager performs numerous calculations per second, but at its heart the QMan's logic is fairly simple. It's operation follows this procedure:

- New submissions are checked to make sure the request is possible and to determine two important times: the earliest time the new group can be started and continue without interruption, and the latest time it can start and still complete.

- Any group that has not started within its time window is removed from the queue.

- Images on the queue are sorted by priority, group submission time, and their order within their group.

- The image highest on the list that can be observed now is done next. This will skip any images that aren't ready to go due to altitude limits, time constraints, etc.

- The priority of an observation that has been started is raised to ensure it will finish.

- Some other rules are followed to ensure we never start something we are unable to finish, etc.

Although this is fairly simple it works quite well for our needs, where priority is more important than minimizing slew times. It has been in use for a few years. There are plans to consider slew times in the optimization as well, particularly for lower priority objects, and to try schemes that involve optimally laying out the remaining night given the current objects on the queue.

As the queue is processed, the status of an individual image changes, following the flow shown in Figure 3. Observers may hold or cancel observations, as well as change their priority. Status updates are displayed in our queue viewer and on our coverage tool. 


\subsection{Queue Viewer}

While the procedure above only picks the next image to be observed, it is applied iteratively, along with slew and overhead time models, to predict how the whole night would unfold if nothing were added to or removed from the queue. This is not needed for telescope operation but allows the night to be previewed by observers and adjusted if desired. The queue viewer (QView) provides the interface for this functionality (see Figure 4).

\section{AUTOMATIC FOCUS CORRECTION}

Besides the high-level automation covered above, there is a lot of low-level automation occurring to make our observations possible. One important time and money saver has been automatic focus correction. It automatically adjusts focus to compensate for temperature-induced expansion and contraction and position-dependent tube flexure. It has made it possible for us to only focus once or twice a night, much less than without it, and means we don't need a dedicated focus sensor.

The temperature corrections are made by reading the temperatures of the struts or tube and applying a calibrated temperature correction coefficient. The flexure corrections for a given telescope position are read from a predefined flexure correction map (see Figure 5). The temperature coefficient and map of corrections as a function of altitude and azimuth are derived from data that is usually acquired on bright Moon nights or nights severely affected by clouds. When we used to focus more often the data could also be derived from the usual focus runs, but since we no longer need to focus very often that's no longer feasible. In other words, automatic focus correction has greatly reduced the time spent focusing. We are constantly looking for ways to improve our efficiency.

\section{CONCLUSIONS}

Thanks to this higher-level automation, our telescopes are becoming increasingly autonomous from target selection to data processing. While the pipeline automatically finds most known objects with small uncertainties, we continue to rely on observers to confirm, vet, and submit NEO candidates and follow-up, a task that is very important and cannot be done effectively by a machine. However, observers now spend far less time actually running the telescopes, and instead can concentrate on examining the data and obtaining results. In fact, our observers have recently started operating two of our telescopes at once, allowing more NEOs to be followed up with the same manpower.

Acknowledgements: Thanks to Scott Swindell, Chris Johnson, Greg Stafford, and others at Steward Observatory for telescope control systems and other automation, as well as all of the Steward Observatory employees that maintain our telescopes. Our increasing automation really puts the telescopes through their paces. The Catalina Sky Survey is supported by NASA's Near-Earth Object Observations Program under grant \#NNX15AF79G.

\section{REFERENCES}

Catalina Real-time Transient Survey 2017, The Catalina Surveys Data Release 2 (Pasadena, CA: Caltech), http://nessi.cacr.caltech.edu/DataRelease/

Center for NEO Studies 2018, Discovery Statistics by Survey (Pasadena, CA: JPL), https://cneos.jpl. nasa.gov/stats/site_all.html

Stokes, G. H., Yeomans, D. K., Bottke, W. F., et al. 2003, NASA Report of the Near-Earth Object Science Definition Team (2003), 112, https://cneos.jpl.nasa. gov/doc/SDT_report_2003.html

The Minor Planet Center 2018, Summary of PHA and NEA Discoveries by Discoverers (Cambridge, MA: Harvard), https://www.minorplanetcenter. net/iau/lists/YearlyBreakdown.html 